\title{
Procesos pedagógicos y uso de tecnología en el aula ${ }^{1}$
}

\section{Teaching processes and technology use in the classroom}

\author{
Marco Antonio VILLALTA PAÚCAR ${ }^{1}$, Angélica GUZMAN ${ }^{2}$ y Miguel \\ NUSSBAUM $^{2}$ \\ ${ }^{1}$ Universidad de Santiago de Chile y ${ }^{2}$ Pontificia Universidad Católica de Chile
}

Recibido: Noviembre 2013

Aceptado: Diciembre 2013

\begin{abstract}
Resumen
Los soportes computacionales han diversificado las posibilidades de interactividad de la enseñanza en sala de clase. Los usos del computador en actividades educativas tales como juegos colaborativos, sistemas de retroalimentación de respuesta o participación simulada, entre otros, ponen en evidencia las diversas alternativas que tiene el uso de esta tecnología al interior del proceso educativo desarrollado en el aula. En este contexto, el propósito del artículo es evidenciar el aporte que genera la mediación computacional para los procesos pedagógicos implementados en la sala de clase de la educación escolar formal. Para ello se realizó un análisis bibliográfico que permitió identificar dos configuraciones de los procesos pedagógicos generados, la Inductiva y la Deductiva. Se analizaron y sistematizaron las actividades de enseñanza mediadas por el computador asociadas a ambos procesos. Entre los resultados se delimitan las ventajas del uso de la tecnología en este escenario, destacándose como principales aportes la estructuración y profundización gradual de los contenidos, el fortalecimiento de los procesos de colaboración, la diversificación de los entornos de aprendizaje, y el incremento de la participación estudiantil.
\end{abstract}

Palabas clave: proceso de enseñanza, enseñanza asistida por ordenador, organización de la clase, informática educativa, innovación pedagógica.

\begin{abstract}
Computer supports have diversified the possibilities for interactivity in classroom teaching. Collaborative games, response feedback systems and simulated participation are indicative of the wide range of activities for which these technologies have found application in classroom education processes. In this article, evidence is offered for the contributions made to classroom pedagogical processes by computer mediation. The bibliographic analysis conducted permitted the identification of 2 pedagogic processes generated, an inductive and a deductive one, were identified. The computer-mediated teaching activities associated with each process in this literature were then classified and analyzed. Finally, inferences were drawn regarding the advantages of computer mediation in this scenario, being the main contributions, structuring
\end{abstract}

${ }^{1}$ Este trabajo fue financiado por el CONICYT-FONDECYT del gobierno de Chile $\mathrm{N}^{\circ} 1100309$. 
and gradual deepening of subject content, the strengthening of collaboration processes, the diversification of learning environments and increased student participation.

Keywords: teaching methods, computer assisted instruction, class organization, computer uses in education, teaching method innovations.

Tradicionalmente, y aún en el siglo XXI, se pueden encontrar procesos de enseñanza en el aula que privilegian una estructura de comunicación unidireccional generada desde el profesor (Pontefract \& Hardman, 2005). Esta lógica centrada en la transmisión de contenidos, no ha logrado demostrar su eficacia para asegurar aprendizajes (Shechtman \& Leichtentritt, 2004). Por su parte, el énfasis en una estructura de comunicación mayormente interactiva ha revolucionado la concepción tradicional de enseñanza y aprendizaje; relevando, entre otros aspectos, el carácter cooperativo y colaborativo del proceso educativo (Vermette, Harper \& DiMillo, 2004), el uso de soportes computacionales para promover la participación (Sherman, 2000), y la dimensión afectiva como condición generadora de un clima emocional adecuado para el aprendizaje (Shechtman \& Leichtentritt, 2004).

Estas propuestas parten del supuesto que el aprendizaje es el resultado de una interacción social con fines educativos, vinculando participación y aprendizaje (Swan, Van Hooft, Kratcoski \& Unger, 2005; Panitz, 1999).

En este marco, el constante desarrollo tecnológico de la sociedad moderna, la democratización de la educación a través de la tecnología de educación a distancia (Larreamendy-Joerns \& Leinhardt, 2006), la rapidez y abundancia de la información, entre otros aspectos, requieren y desarrollan en los estudiantes habilidades cognitivas sustentadas en procesos interactivos y colaborativos (Yan, 2008; Greenfield, 2009). Desde estos antecedentes, el dilema en innovación educativa no consiste en utilizar la tecnología computacional en el trabajo de aula, sino en lograr su adecuada integración a los procesos educativos (Dillenbourg, 2008; Hogarth, 2008; Guzman \& Nussbaum, 2009).

La incorporación de soportes tecnológicos al aula ha permitido mejorar la transmisión y construcción colaborativa de conocimiento tanto en el espacio virtual (Cobos \& Pifarré 2008) como en la relación cara a cara (Swan, Van Hooft, Kratcoski, \& Unger, 2005; Cortez, Nussbaum, Woywood \& Aravena, 2008), a la vez que ha diversificado los procedimientos de enseñanza con el incremento de la interactividad para el logro de objetivos de aprendizaje (Cortez Nussbaum, Woywood \& Aravena, 2008; Balram \& Dragicevic, 2008).

A pesar de los avances en relación con las propuestas de uso educativo del computador, tanto en estudios de habla hispana como inglesa, no se encuentra consenso en categorías conceptuales que aborden los productos pedagógicos asociados a su integración en el aula (Collazos, Guerrero, Pino, Renzi, Klobas, Ortega, Redondo \& Bravo, 2007; Bravo, Redondo, Verdejo \& Ortega, 2008), precisando el impacto formativo que ofrecen las tecnologías computacionales a los procesos de enseñanza. 
El objetivo del presente artículo es analizar los procesos pedagógicos que, en la sala de clases de la educación escolar formal, pueden ser potenciados por la mediación computacional.

A continuación se describe la metodología utilizada; seguidamente se presentan los resultados del análisis del proceso pedagógico mediado por el computador en la sala de clases; luego sigue la discusión; y, finalmente se presenta las conclusiones del trabajo realizado.

\section{Metodología}

El método de estudio es de tipo cualitativo desarrollando el análisis de contenido de un conjunto de artículos científicos publicados en revistas indexadas y de acceso abierto entre los años 2000 y 2009.

\section{Selección de las fuentes de estudio.}

Las fuentes de estudio fueron artículos científicos seleccionados desde nueve bases de datos a partir de la aplicación de dos filtros de palabras clave vinculadas al objetivo del estudio. Se privilegian los estudios de habla inglesa para delimitar el campo de estudio a los aspectos metodológicos de uso de tecnología computacional en el aula sin complejizarlos con las categorías didácticas desarrollados en estudios de habla hispana. El primer filtro de términos permitió seleccionar textos que integraran las siguientes palabras clave (key words): Computer, Technology, Teaching y Methods. El segundo filtro consideró la explicitación en los documento de los siguientes conceptos: Classroom, Interactive y Participatory. En la Tabla 1 se presenta una síntesis del trabajo realizado, indicando las Bases de datos consultadas, Filtros de selección y Número de artículos analizados.

\begin{tabular}{|c|c|c|c|}
\hline Bases de Datos & $\begin{array}{c}\text { Primer Filtro } \\
\text { Key words: } \\
\text { Computer, } \\
\text { Technology } \\
\text { Teaching, Methods. }\end{array}$ & $\begin{array}{l}\text { Segundo Filtro } \\
\text { Conceptos: } \\
\text { Classroom } \\
\text { Interactive } \\
\text { Participatory. } \\
\end{array}$ & $\begin{array}{c}\text { Total de } \\
\text { textos }\end{array}$ \\
\hline Academic Search Premier database. & 12 & 3 & \\
\hline Education Research Complete database & 6 & 2 & \\
\hline ERIC Database & 15 & 10 & \\
\hline ProQuest Education Journals database & 4 & 0 & \\
\hline ProQuest Humanities & 2 & 0 & 49 \\
\hline ProQuest Psychology Journals & 1 & 0 & \\
\hline Research Library Core database & 1 & 0 & \\
\hline Scholar google & 7 & 3 & \\
\hline Web of Science & 49 & 31 & \\
\hline
\end{tabular}

Tabla 1. Síntesis de textos analizados referidos a Métodos de enseñanza mediados por soportes computacionales en sala de clases. 


\section{Estrategia de análisis de información.}

Para realizar el análisis se emplearon principios del Análisis de Contenido (Ricoy, Feliz y Sevillano, 2012) en la generación de un sistema de categorías primarias, teóricamente fundadas que orientaron la organización de la información de los artículos y la formación de sub-categorías. Este permitió desarrollar un análisis de contenido centrado en el contraste y en la construcción de categorías que describiesen exhaustivamente el proceso pedagógico, a partir de indicadores comunes en los textos (Pasquale \& Meunier, 2003). El análisis concluyó cuando la muestra se saturó teóricamente, es decir, al momento que no aparecieron textos que enriqueciesen la generación de nuevas categorías (Flick, 2006; Glaser \& Holton, 2004).

Las primeras categorías se relacionaron con la organización temporal del proceso pedagógico, determinando tres fases configuradoras de la clase: Inicio, Desarrollo y Cierre (Sinclair \& Coulthard, 1975; Mehan, 1979; McFarland \& Bender-deMoll, 2004). De modo operativo, para el presente estudio, estas fases se definieron de la siguiente forma:

a) Inicio. Referida a la explicitación del propósito y a la organización de las actividades de la clase. Esta fase corresponde a los primeros minutos de la sesión.

b) Desarrollo. Centrada en la presentación y discusión de contenidos disciplinares. Éste es el segmento central de la clase y ocupa la mayor cantidad de tiempo del trabajo de aula.

c) Cierre. Focalizada en levantar conclusiones de las actividades realizadas en la clase. Generalmente ocupa los últimos minutos de la sesión.

Al interior de cada fase, se desarrollan actividades disciplinares específicas que organizan el proceso pedagógico. Se trata de guiones de acción que siguen profesor y alumnos, para alcanzar los objetivos educativos (Dillenbourg, 2002).

Con independencia de la fase en que se presenten, estos guiones de acción fueron caracterizados a partir de dos focos: el objetivo de aprendizaje y su relación con una estrategia metodológica específica.

El objetivo de aprendizaje se define como el propósito que persigue el profesor en relación con el dominio que los estudiantes deben alcanzar sobre determinados contenidos educativos. En algunos casos el propósito es que los estudiantes fortalezcan la asimilación de contenidos (Panitz, 1999; Crouch \& Mazur, 2001; Burns, 2005); mientras que en otros se pretende la construcción de diagramas y argumentaciones (Van Amelsvoort, Andriessen \& Kanselaar, 2007; Peled, Meron \& Rota, 2007), o la negociación y el consenso en las respuestas (Colella, 2000; Zurita \& Nussbaum, 2004a, 2004b).

La estrategia metodológica está referida a la organización de las actividades del profesor y de los alumnos durante la clase, la que puede adquirir distintos niveles de interactividad, dependiendo de las orientaciones pedagógicas que la sustenten (Ang, Avni \& Zaphiris, 2008). Esta puede ser pre-estructurada, cuando se define a priori la 
forma y el orden de la participación; o regulada cuando se permite la participación emergente de los alumnos (Dillenbourg, 2002).

En consideración de ambos criterios, Objetivo de la lección y Estrategia metodológica, se delimitan dos configuraciones de los Procesos Pedagógicos que se desarrollan en el aula: el Proceso Deductivo y el Proceso Inductivo.

En el Proceso Pedagógico Deductivo, las actividades de enseñanza tienen como propósito que el estudiante asimile y aplique los contenidos a una situación de aprendizaje; la estrategia metodológica se focaliza en la estructuración de la acción del estudiante; y al término de la clase el profesor verifica el proceso realizado y se completa la información a través de la retroalimentación.

En el Proceso Pedagógico Inductivo, las actividades de enseñanza tienen como propósito que el estudiante diseñe, construya, aplique y argumente su respuesta a una situación de aprendizaje. La estrategia metodológica se inicia con la presentación de una situación problema, y termina con la formalización comprehensiva de los aprendizajes esperados.

En la Tabla 2 se describen estos Procesos Pedagógicos, indicando, en la primera columna, las tres fases temporales de la lección, en la segunda columna se describen las categorías del Proceso Pedagógico Deductivo y, en la última columna, se describen las categorías del Proceso Pedagógico Inductivo.

\begin{tabular}{|l|l|l|}
\hline Fase & \multicolumn{1}{|c|}{ Categorías del Proceso Deductivo } & \multicolumn{1}{c|}{ Categorías del proceso Inductivo } \\
\hline \multirow{5}{*}{ Inicio } & $\begin{array}{l}\text { Información estructurante. } \\
\text { Actividades del profesor que } \\
\text { comunican a los estudiantes los } \\
\text { objetivos de la clase y las acciones a } \\
\text { desarrollar. }\end{array}$ & $\begin{array}{l}\text { Situación problema. Actividades del } \\
\text { profesor que presentan a los estudiantes } \\
\text { una situación disciplinar específica a } \\
\text { resolver. }\end{array}$ \\
\hline \multirow{5}{*}{ Desarrollo } & $\begin{array}{l}\text { Aplicación de procedimiento. } \\
\text { Actividades de los estudiantes cuyo } \\
\text { propósito es ejercitar y aplicar la } \\
\text { información previamente entregada, } \\
\text { en situaciones estructuradas de } \\
\text { aprendizaje. }\end{array}$ & $\begin{array}{l}\text { Construcción de la respuesta. } \\
\text { Actividades de los estudiantes cuyo } \\
\text { propósito es elaborar, explorar y evaluar } \\
\text { estrategias de respuesta, a la situación } \\
\text { disciplinar presentada al inicio de la } \\
\text { clase. }\end{array}$ \\
\hline & $\begin{array}{c}\text { Verificación de la información. } \\
\text { Actividades del profesor } \\
\text { orientadas a retroalimentar y } \\
\text { completar la información trabajada } \\
\text { en la clase. }\end{array}$ & $\begin{array}{l}\text { Formalización disciplinar. } \\
\text { Actividades de profesor y alumnos } \\
\text { orientadas a transferir la estrategia } \\
\text { construida a los conceptos formales de } \\
\text { la disciplina. }\end{array}$ \\
\hline
\end{tabular}

Tabla 2. Categorías del proceso pedagógico en sala de clase 


\section{Resultados}

Los soportes computacionales aportan a los procesos pedagógicos desarrollados en el aula, sean estos deductivos o inductivos. A continuación se presentan y analizan algunas actividades realizadas en ambos procesos, deductivo e inductivo, con el uso de mediación computacional.

Proceso Pedagógico Deductivo en sala de clase, mediado por soportes computacionales

Se consideran aquí los procedimientos interactivos que refuerzan la adquisición de información por parte de los estudiantes, a través de entornos virtuales adaptados a diversos estilos de aprendizaje (Mayo, 2009).

\section{Fase de Inicio}

Algunas actividades del profesor asociadas a esta categoría son:

- Información instruccional. Refiere a la entrega de información conceptual pertinente al tema a trabajar en clase. Por ejemplo, en la propuesta Peer Instruction de Crouch y Mazur (2001), se realizan presentaciones breves en pantalla de conceptos centrales de un contenido específico.

- Enunciado de la actividad. Consiste en la presentación de situaciones aplicativas en la clase. En este caso el soporte computacional motiva y organiza la participación de los alumnos (Roschelle, Penuel \& Abrahamson, 2004; Nussbaum, Alvarez, McFarlane, Gomez, Claro \& Radovic, 2009).

\section{Fase de Desarrollo}

En esta categoría se evidencian tres ejes distintos de actividades: a) Asimilación de información; b) Ejecución; y, c) Retroalimentación.

a) Asimilación de información. Considera los procesos individuales y grupales de comprensión y desarrollo de acciones a realizar. Estos pueden ser:

- Elaboración Individual: donde el alumno aplica la información disciplinar, respondiendo al problema expuesto. Por ejemplo: elección de una respuesta desde un conjunto de alternativas (Crouch \& Mazur, 2001); ejercitación con diferentes niveles de complejidad y sistema de autorregulación (Rosas, Nussbaum, Cumsille, Marianov, Correa, Flores, Lopez, Rodriguez \& Salinas, 2003); o, elaboración colaborativa de una respuesta a partir de información previamente entregada (Linnell, Anderson, Fridley, Hinckle \& Razmov, 2007). 
- Elaboración Grupal: actividades que fomentan el diálogo en pequeños grupos de alumnos, para elaborar respuestas consensuadas en la actividad solicitada. Por ejemplo: negociación de respuesta (Infante, Hidalgo, Nussbaum, Alarcón \& Gottlieb, 2009); u, organización en la toma de decisiones (Zurita \& Nussbaum, 2007).

b) Ejecución. Actividades de aplicación de decisiones asumidas, a las situaciones a resolver presentadas en la clase. Éstas pueden ser:

- Multiple Choise questions. Consiste en la elección de respuesta a una pregunta con múltiples alternativas. Por ejemplo, en la evaluación del aprendizaje de contenidos específicos (Crouch \& Mazur, 2001; Samson, Teasley, Van Der Pluijm \& Knoop, 2006), aplicable a una amplia variedad de áreas disciplinares (Roschelle, Penuel \& Abrahamson, 2004; Beatty, Leonard, Gerace \& Dufresne, 2005; Beal, Qu \& Lee, 2008).

- Strategy development. Situación en que los estudiantes aplican la información previamente entregada, para desarrollar una respuesta disciplinar. Por ejemplo, el diseño computacional de acciones a ser implementadas en el laboratorio (Dantas \& Kemm, 2008).

- Concept Arrangement. Consistente en la organización de una situación de acuerdo a criterios anteriormente entregados. Por ejemplo: Organización de hechos históricos con uso de software (Wetzel, Foulger \& Williams, 2008).

c) Retroalimentación. Actividades que retroalimentan las respuestas de los alumnos de modo individual y/o grupal. Éstas pueden ser:

- Comprobación del dominio de contenidos disciplinares entregados (Fies \& Marshall, 2006; Penuel, Boscardin, Masyn \& Crawford, 2007; Crouch \& Mazur, 2001; Beatty Leonard, Gerace \& Dufresne, 2005; Samson, Teasley, Pluijm \& Knoop, 2006; Deal, 2007); o, adecuación de la explicación de contenidos al tipo de respuestas dadas por lo alumnos, a través del análisis dialogado de las respuestas sistematizadas por el software (Beal, Qu \& Lee, 2008).

- Corrección de la respuesta, en caso de no estar correcta (Zurita, Nussbaum \& Sharples, 2003; Cortez, Nussbaum, López, Rodríguez, Santelices, Rosas \& Marianov, 2005).

\section{Fase de Cierre}

Referido a las actividades finales de ajuste y transferencia de la información presentada en la clase a otras situaciones donde se pueda aplicar el aprendizaje logrado. En esta fase se retroalimenta todo el proceso de la lección, el profesor relaciona los contenidos disciplinares con las actividades realizadas por los alumnos. Por ejemplo: Sistemas computacionales de respuesta, para el ajuste y transferencia de actividades de aprendizaje, (Beatty Leonard, Gerace \& Dufresne, 2005); o, Registro tecnológico de las respuestas (Fies, 2005; Deal, 2007); la mediación tecnológica 
permite el acercamiento continuo entre lo que enseña el profesor y el aprendizaje activo de los alumnos.

El proceso Pedagógico Deductivo mediado por soportes computacionales se presenta sintéticamente en la Tabla 3 . En esta se ejemplifican sólo las actividades más representativas de cada fase y categoría del proceso.

\begin{tabular}{|c|c|c|c|}
\hline Fase & Categorías & Sub-categorías & Ejemplos de Actividades \\
\hline Inicio & $\begin{array}{l}\text { Información } \\
\text { estructurante }\end{array}$ & & $\begin{array}{l}\text { - Información instruccional (Crouch \& } \\
\text { Mazur, 2001) }\end{array}$ \\
\hline \multirow{3}{*}{ Desarrollo } & \multirow{3}{*}{$\begin{array}{l}\text { Aplicación de } \\
\text { procedimiento }\end{array}$} & $\begin{array}{l}\text { Asimilación de la } \\
\text { información }\end{array}$ & $\begin{array}{l}\text { - Ejercitación con diferentes niveles de } \\
\text { complejidad (Rosas, Nussbaum, Cumsille, } \\
\text { Marianov, Correa, Flores, Lopez, } \\
\text { Rodriguez \& Salinas,2003). } \\
\text { - Elaboración Grupal (Zurita \& Nussbaum, } \\
\text { 2004b, 2007). }\end{array}$ \\
\hline & & Ejecución & $\begin{array}{ll}\text { - } & \text { Multiple Choise questions (Crouch \& } \\
\text { Mazur, 2001) } \\
\text { - } \quad \text { Strategy development (Dantas \& Kemm, } \\
\text { 2008). } \\
\text { - Concept Arrangement (Wetzel, Foulger \& } \\
\text { Williams, 2008). }\end{array}$ \\
\hline & & Retroalimentación & $\begin{array}{l}\text { - Comprobación del dominio disciplinar } \\
\text { (Fies \& Marshall, 2006). } \\
\text { - Corrección de la respuesta (Zurita, } \\
\text { Nussbaum \& Sharples, 2003). }\end{array}$ \\
\hline Cierre & $\begin{array}{l}\text { Verificación de } \\
\text { información }\end{array}$ & & $\begin{array}{l}\text { - } \quad \text { Ajuste y transferencia de actividades de } \\
\text { aprendizaje (Beatty Leonard, Gerace \& } \\
\text { Dufresne, 2005) }\end{array}$ \\
\hline
\end{tabular}

Tabla 3. Síntesis del Proceso Pedagógico Deductivo con mediación computacional

Proceso Pedagógico Inductivo en sala de clase, mediado por soportes computacionales

Se consideran aquí los procedimientos interactivos que apuntan a promover aprendizaje a través de la inmersión del estudiante en la situación de aprendizaje (Dede, 2009), con el propósito de analizar y resolver situaciones problema propuestas (Shaffer \& Gee, 2005; Kiili, 2007).

\section{Fase de Inicio}

Las actividades del profesor asociadas a esta fase se agrupan en la siguiente categoría:

- Presentación de la situación problema: El profesor expone el problema y los aspectos a considerar para resolverlo (Colella, 2000). El soporte 
computacional favorece la inmersión de todos los estudiantes en una determinada tarea o misión (Dickey, 2006; Amory, 2007; Lim, 2008). Por ejemplo, para el caso de la economía, presentación de una situación de mercado simulada, en la que los estudiantes dirigen una empresa que compite para ganar dicho mercado (Kiili, 2007).

\section{Fase de Desarrollo}

En esta fase las actividades se agrupan en tres categorías: a) Inmersión y negociación de la estrategia; b) Construcción y ejecución de la estrategia; y, c) Reflexión.

a) Inmersión y negociación de la estrategia. Actividad en que el estudiante se involucra en la situación presentada, dialogando con pares, explorando herramientas y ensayando respuestas tentativas. Por ejemplo: Exploración de los recursos entregados en clase, para crear juegos de computador que promueva el desarrollo de habilidades sociales entre los estudiantes (Robertson \& Howells, 2008). Los soportes tecnológicos favorecen actividades como:

- Estructuración de la respuesta a través de sistemas de registro y secuenciación de la participación individual dentro del grupo (Nussbaum, Alvarez, Mitnik, Gomez, Radovic, Claro, Lopez \& Rodriguez, 2008; Nussbaum, Alvarez, McFarlane, Gomez, Claro \& Radovic, 2009), favoreciendo el proceso de participación colaborativa y la negociación (Beers, Kirschner, Boshuizen \& Gijselaers, 2007), y construyendo una solución al problema planteado en, por ejemplo, situaciones de video juego (Infante, Weitz, Reyes \& Nussbaum, 2009).

- Diálogo argumentado entre las diferentes perspectivas de los participantes (Ravenscroft, 2007), fortaleciendo la constitución de comunidad de aprendizaje en, por ejemplo, el mundo virtual donde se ha presentado la situación problema (Jong, Shang, Lee \& Lee, 2009); o, en el trabajo de Colella (2000), donde se pide a los alumnos sistematizar las interacciones desarrolladas, para discernir los mecanismos de transmisión de un virus,

- Utilización de herramientas para profundizar el conocimiento, por ejemplo, el uso del computador para indagar, sistematizar y registrar el conocimiento construido colaborativa o individualmente (Syh-Jong, 2007).

b) Construcción y aplicación de estrategias. Actividades de colaboración entre todos los participantes para resolver la situación problema. Éstas pueden ser:

- Games for developing abilities. Actividades cuyas reglas predefinidas de interacción permiten a los participantes desarrollar habilidades para abordar situaciones específicas presentadas. Por ejemplo: Juegos epistémicos (Shaffer, 2006), en los que la resolución de la situación problema requiere de la aplicación de conocimientos específicos, o de la ejercitación de habilidades viso-espaciales (Orvis, Horn \& Belanich, 2008). 
- $\quad$ Schematic representation. Actividades de representación gráfica de conceptos u opiniones referidas a la situación problema. Esto puede ser aplicado para promover el análisis argumentativo de problemas que no tienen respuesta única. Por ejemplo, Van Amelsvoort, Andriessen \& Kanselaar (2007), solicitan a los estudiantes la construcción de un diagrama que refleje la opinión que estos tienen sobre el tema de los organismos genéticamente modificados;

- $\quad$ Proyectos de participación. Actividades de coordinación de diversas acciones para resolver la situación problema planteada. Por ejemplo, la propuesta de Robertson \& Howells (2008), en la que los estudiantes construyen juegos por computador, para promover la creatividad y la participación; la toma de decisiones para editar presentaciones interactivas que promuevan la educación intercultural (Thurston, 2004); o, el aprendizaje sobre equilibrio ecológico y la promoción de valores ciudadanos a través de la participación en un mundo virtual (Lim, 2008; Susaeta, Jimenez, Nussbaum, Gajardo, Andreu \& Villalta, 2010).

c) Reflexión. Son actividades donde los estudiantes analizan las causas y consecuencias de sus acciones individuales y colectivas para la resolución del problema. Las actividades pueden estar referidas a:

- Procesos autónomos. Donde los estudiantes analizan sus acciones sin la participación del profesor; por ejemplo, en el aprendizaje colaborativo de conceptos de herencia genética mendeliana, a través de la participación simulada mediada por soportes computacionales portátiles (Klopfer, Yoon \& Rivas, 2004).

- Procesos mediados por el profesor. Donde el profesor participa en la reflexión de las estrategias aplicadas por los estudiantes, entregando criterios para fortalecer el análisis. Por ejemplo, las actividades dialogales sobre el uso adecuado de un determinado saber disciplinar, para dar respuesta a una situación problema (Nussbaum, Alvarez, McFarlane, Gomez, Claro \& Radovic, 2009); el análisis de estrategias de acción empleadas para resolver misiones en el mundo virtual para el cuidado de un entorno ecológico determinado (Lim, 2008; Susaeta, Jimenez, Nussbaum, Gajardo, Andreu \& Villalta, 2010); o, la vinculación de los aspectos virtuales de simulación con la realidad (Jong, Shang, Lee \& Lee, 2009).

\section{Fase de Cierre}

Refiere a las actividades desarrolladas tanto por el profesor como por los estudiantes, con el propósito de explicitar los aprendizajes logrados en la experiencia educativa, formalizando disciplinarmente el proceso realizado y presentando la funcionalidad de las estrategias implementadas para la solución del problema planteado. Las actividades asociadas a esta categoría son: 
- Diálogo en torno al aprendizaje. Consiste en la discusión generada entre los participantes en torno a la experiencia de aprendizaje vivenciada, lo que permite lograr la reflexión crítica del conocimiento efectivamente construido (Kiili, 2007). Por ejemplo, en la situación simulada que genera competencia empresarial para ganar un mercado específico (Kiili, 2007); en el caso del comportamiento económico de la bolsa de valores (Aldinger, Kopf, Scheele \& Effelsberg, 2005); o, en el análisis de logro de competencias para el trabajo grupal, a través de autorreporte (Cortez, Nussbaum, Woywood \& Aravena, 2008).

- Presentación del trabajo realizado. Referido a la presentación que realizan los alumnos al profesor y a la clase completa, en torno al proceso y al producto del aprendizaje generado. Por ejemplo, la presentación de cuentos construidos interactivamente, a través de computadores conectados en red (Carbonaro, Cutumisu, Duff, Gillis, Onuczko, Siegel, Schaeffer, Schumacher, Szafron \& Waugh, 2008); o, la presentación de juegos computacionales construidos por los estudiantes (Robertson \& Howells, 2008; Kirriemuir \& McFarlane, 2004). Esta fase también se relaciona con la presentación de lo aprendido en la aplicación práctica de conceptos disciplinares. Por ejemplo, aplicación de conceptos de cinemática para lograr el movimiento de un robot (Mitnik, Recabarren, Nussbaum \& Soto, 2009).

El proceso Pedagógico Inductivo mediado por soportes computacionales se presenta sintéticamente en la Tabla 4. En ésta se ejemplifican sólo las actividades más representativas de cada fase y categoría del proceso.

\begin{tabular}{|c|c|c|c|}
\hline Fase & Categorías & $\begin{array}{l}\text { Sub- } \\
\text { categorías }\end{array}$ & Ejemplos de Actividades \\
\hline Inicio & $\begin{array}{l}\text { Situación } \\
\text { Problema }\end{array}$ & & $\begin{array}{l}\text { • Inmersión en una tarea o misión (Lim, 2008; Kiili, } \\
\text { 2007) }\end{array}$ \\
\hline \multirow{3}{*}{ Desarrollo } & \multirow{3}{*}{$\begin{array}{l}\text { Construcción } \\
\text { de respuesta }\end{array}$} & $\begin{array}{l}\text { Negociación } \\
\text { de estrategia }\end{array}$ & $\begin{array}{l}\text { - Negociación de respuesta (Beers, Kirschner, } \\
\text { Boshuizen \& Gijselaers, 2007). } \\
\text { - Diálogo argumentado (Ravenscroft, 2007). } \\
\text { - Profundización en el conocimiento (Syh-Jong, 2007). }\end{array}$ \\
\hline & & $\begin{array}{l}\text { Construcción } \\
\text { y ejecución de } \\
\text { estrategia }\end{array}$ & $\begin{array}{l}\text { - Games for developing abilities (Shaffer, 2006). } \\
\text { - Schematic representation (Van Amelsvoort, } \\
\text { Andriessen \& Kanselaar, 2007). } \\
\text { - Proyectos de participación (Robertson \& Howells, } \\
\text { 2008) }\end{array}$ \\
\hline & & Reflexión & $\begin{array}{l}\text { - Procesos autónomos (Klopfer, Yoon \& Rivas, 2004) } \\
\text { - Procesos mediados (Nussbaum, Alvarez, McFarlane, } \\
\text { Gomez, Claro, \& Radovic, 2009). }\end{array}$ \\
\hline Cierre & $\begin{array}{l}\text { Formalización } \\
\text { disciplinar }\end{array}$ & & $\begin{array}{l}\text { - Diálogo en torno al aprendizaje (Aldinger, Kopf, } \\
\text { Scheele \& Effelsberg, 2005). } \\
\text { - Presentación del trabajo realizado (Robertson \& } \\
\text { Howells, 2008; Kirriemuir \& McFarlane, 2004). }\end{array}$ \\
\hline
\end{tabular}

Tabla 4. Síntesis del Proceso Pedagógico Inductivo con mediación computacional 


\section{Discusión}

El análisis realizado indica que el uso del computador en la sala de clases participa de los procesos pedagógicos, ya sean estos deductivos o inductivos. Estudios realizados en sala de clase de educación Básica en Santiago de Chile demuestran que el uso de recursos computacionales en el aula favorecen la participación activa de los estudiantes cuando la herramienta tecnológica logra adaptarse a las necesidades de uso del estudiante (Alcoholado, Nussbaum, Tagle, Gomez, Denardin, Susaeta, Villalta, Toyama, 2012); o cuando logra estructurar un patrón de acciones para el trabajo colaborativo (Caballero, van Riesen, Alvarez, Nussbaum, De Jong, 2014), favoreciendo por una parte, la retroalimentación inmediata a la participación y, por otra parte, el andamiaje que facilita y permite el trabajo colaborativo.

En el Proceso Pedagógico Deductivo, la relación frontal de presentación de contenidos es importante, pero no es suficiente para completar la asimilación de un contenido por parte de los estudiantes. El logro de fluidez en esta habilidad cognitiva se alcanza de mejor forma integrando actividades de visualización y ejercitación de procedimientos, junto con actividades de retroalimentación de respuesta; lo que se logra con un tipo de interactividad generada por la tecnología pertinente a este proceso.

Los soportes computacionales potencian el rol docente en este aspecto, otorgándole herramientas que favorecen la organización lógica de la información. Dicho de otro modo, le permiten trabajar con guiones de acción pre-estructurados, que facilitan la presentación y transmisión del contenido disciplinar.

Por su parte, el Proceso Pedagógico Inductivo requiere de entornos de aprendizaje que faciliten al profesor y a sus estudiantes diseñar y aplicar el conocimiento a las situaciones didácticas planteadas en la clase. Los soportes computacionales han potenciado el proceso pedagógico inductivo generando una mediación que diversifica el diseño de tales situaciones didácticas para diferentes procesos de aprendizaje en la sala de clase, a la vez que favorece la aplicación y evaluación de estrategias de acción construidas en el trabajo colaborativo de los estudiantes, que potencia la motivación intrínseca y el aprender haciendo.

Tanto los procesos pedagógicos Deductivos como los Inductivos preexisten al uso del computador en el aula, y como queda demostrado, existen soportes computacionales que potencian a ambos. El uso de soportes computacionales agrega al Proceso Pedagógico Deductivo elementos como: mayor interactividad en la relación comunicativa de sala de clase ampliando los canales de comunicación -visual y verbal-, enriqueciendo así los entornos de aprendizaje; retroalimentación inmediata, tanto a la acción de enseñanza del profesor como a las actividades de aprendizaje de los alumnos; y, estructuración y graduación interactiva de la transmisión del contenido, ajustado al proceso de aprendizaje de los alumnos.

La mediación tecnológica potencia también las actividades del Proceso Pedagógico Inductivo en aspectos como: diversificación de los entornos de aprendizaje a través de, por ejemplo, mundos virtuales cuya organización de elementos en misiones integran diversas situaciones didácticas donde los estudiantes son desafiados a explorar, construir y aplicar estrategias de solución; favorecimiento de la participación directa de 
los estudiantes, a través de, por ejemplo, juegos interactivos o de Participación Simulada, que promueven la inmersión y compromiso con la actividad; y, la estructuración del proceso colaborativo de los estudiantes, a través de, por ejemplo, sistemas de comunicación que organizan los turnos de participación y toma de decisiones, estimulando el desarrollo de habilidades para el trabajo en equipo, junto al aprendizaje de contenidos.

\section{Conclusiones}

La mediación tecnológica fortalece las actividades interactivas de la sala de clase asegurando operatividad, dado que su funcionamiento incorpora procesos establecidos previamente que favorecen la participación de los alumnos. Dicha mediación introduce nuevos recursos para la enseñanza que diversifican las actividades de estudiantes y profesor, favorece la participación directa de los estudiantes, estructura los procesos de colaboración y cuenta con mecanismos de seguimiento y retroalimentación de los propósitos previamente establecidos. En tal sentido, los soportes computacionales permiten a los estudiantes el aprendizaje disciplinar y colaborativo, optimizando la estructuración de los procesos pedagógicos del aula y promoviendo el desarrollo de competencias para el trabajo en equipo.

En el Proceso Pedagógico Deductivo, el uso de tecnología ofrece la posibilidad de profundizar en los contenidos de enseñanza, a través de actividades graduadas en términos de su complejidad, lo que permite adaptarlas al ritmo de aprendizaje de cada estudiante. Complementariamente, el seguir una lógica deductiva en el proceso pedagógico requiere que el profesor fortalezca su rol como mediador entre los estudiantes y el contenido disciplinar, fortaleciendo así la organización y secuencia de la información entregada.

En el Proceso Pedagógico Inductivo los soportes computacionales proporcionan la alternativa de integrar las necesidades, intereses y conocimientos previos de los estudiantes al proceso de enseñanza y aprendizaje que se desarrolla en la sala de clase, generando actividades que promueven la inmersión en las situaciones presentadas. Por otra parte, desde un enfoque inductivo, se fomenta el trabajo colaborativo de los estudiantes, abriendo escenarios para el diseño, aplicación y evaluación de las estrategias de acción requeridas en la correspondiente resolución de problemas.

Los soportes computacionales poseen una estructura de funcionamiento que almacena un estudiado conjunto de acciones pre-establecidas, constitutivas de las buenas prácticas de enseñanza disciplinar, lo que permite orientar la labor docente en los distintos escenarios de aprendizaje generados en el aula. Estos soportes se articulan plenamente tanto con los Procesos Pedagógicos Deductivos como con los Inductivos que puedan ser implementados por el profesor en la clase, permitiéndole consolidar la conducción eficaz de dicho proceso. 


\section{Referencias bibliográficas}

ALCOHOLADO, C., NUSSBAUM, M., TAGLE, A., GOMEZ, F., DENARDIN, F., SUSAETA, H., VILLALTA, M., TOYAMA, K., (2012) One Mouse per Child: Interpersonal Computer for Individual Arithmetic Practice. Journal Computer Assisted Learning 28(4), 295-309, DOI: 10.1111/j.1365-2729.2011.00438.x

ALDINGER, T., KOPF, S., SCHEELE, N., \& EFFELSBERG, W. (2005). Participatory Simulation of a Stock Exchange. In P. Kommers, \& G. Richards, World Conference on Educational Multimedia, Hypermedia and Telecommunications, 3759-3766. Chesapeake, VA: AACE.

AMORY, A. (2007). Game object model version II: a theoretical framework for educational game development. ducational Technology Research and Development, 55, 51-77.

ANG, C., AVNI, E., \& ZAPHIRIS, P. (2008). Linking Pedagogical Theory of Computer Games to Their Usability. International Journal on E-Learning, 7(3) , 533-558.

BALRAM, S., \& DRAGICEVIC, S. (2008). Collaborative spaces for GIS-based multimedia cartography in blended environments. Computers \& Education, 50 , 371-385.

BEAL, C., QU, L., \& LEE, H. (2008). Mathematics motivation and achievement as predictors of high school students' guessing and help-seeking with instructional software. Journal of Computer Assisted Learning, 24, 507-514.

BEATTY, I., LEONARD, W., GERACE, W., \& DUFRESNE, R. (2005). Question Driven Instruction: Teaching Science (Well) with an Audience Response System. In D. A. (Ed.), Audience Response Systems in Higher Education: Applications and Cases. (p. Chapter VII). Hershey, PA: Information Science Publishing.

BEERS, P., KIRSCHNER, P., BOSHUIZEN, H., \& GIJSELAERS, W. (2007). ICTsupport for grounding in the classroom. Instructional Science, 35, 535-556 .

BRAVO, C., REDONDO, M., VERDEJO, M. \& ORTEGA, M. (2008). A framework for process-solution analysis in collaborative learning environments. Int. J. HumanComputer Studies 66, 812-832.

BURNS, M. (2005). Using Incremental Rehearsal to Increase Fluency of Single-Digit Multiplication Facts with Children Identified as Learning Disabled in Mathematics Computation. Education and Treatment of Children, 28(3), 237-249.

CABALLERO, D., VAN RIESEN, S., ALVAREZ, S., NUSSBAUM, M., DE JONG, T. (2014), The Effects of Whole-class Interactive Instruction with Single Display Groupware for Triangles, Computers \& Education, 70, pp 203-211, http://dx.doi.org/10.1016/j.compedu.2013.08.004

CARBONARO, M., CUTUMisU, M., DUFF, H., GILliS, S., ONUCZKO, C., SIEGEL, J., SCHAEFFER, J., SCHUMACHER, A., SZAFRON, D. \& WAUGH, 
K. (2008). Interactive story authoring: A viable form of creative expression for the classroom. Computers \& Education, 51, 687-707.

CARON, T. (2007). Learning Multiplication: The Easy Way. Clearing House: A Journal of Educational Strategies, Issues and Ideas, 80(6) , 278-282.

COBOS, R., \& PIFARRÉ, M. (2008). Collaborative knowledge construction in the web supported by the KnowCat system. Computers \& Education 50, 962-978.

COLELLA, V. (2000). Participatory Simulations: Building Collaborative Understanding through Immersive Dynamic Modeling. The Journal of the Learning Sciences, 9(4) , 471-500.

COLLAZOS, C., GUERRERO, L., PINO, J., RENZI, S., KLOBAS, J., ORTEGA, M., REDONDO, M. \& BRAVO, C. (2007). Evaluating Collaborative Learning Processes using System-based Measurement. Educational Technology \& Society, 10(3), 257-274.

CORTEZ, C., NUSSBAUM, M., LÓPEZ, X., RODRÍGUEZ, P., SANTELICES, R., ROSAS, R. \& MARIANOV, V. (2005). Teachers' support with ad-hoc collaborative networks. Journal of Computer Assisted Learning 21, 171-180.

CORTEZ, C., NUSSBAUM, M., WOYWOOD, G., \& ARAVENA, R. (2008). Learning to collaborate by collaborating: a face-to-face collaborative activity for measuring and learning basics about teamwork. Journal of Computer Assisted Learning, 25(2) , 126-142.

CROUCH, C. \& MAZUR, E. (2001). Peer instruction: ten years of experience and results. American Journal of Physics, 69, 970-977.

DANTAS, A., \& Kemm, R. (2008). A blended approach to active learning in a physiology laboratory-based subject facilitated by an e-learning component. Advances in Physiology Education 32, 65-75.

DEAL, A. (2007). Classroom Response Systems. A Teaching with Technology White Paper. Carnegie Mellon University, Retrieved june 10, 2009, from http://www.cmu.edu/teaching.

DEDE, C. (2009). Immersive Interfaces for Engagement and Learning. Science 323(66-69).

DICKEY, M. (2006). Game Design Narrative for Learning: Appropriating Adventure Game Design Narrative Devices and Techniques for the Design of Interactive Learning Environments. Educational Technology Research and Development, 54(3), 245-263.

DILLENBOURG, P. (2002). Over-scripting CSCL: The risks of blending collaborative. En P. A. (Ed), Three worlds of CSCL. Can we support CSCL (págs. 61-91). Heerlen: Open Universiteit Nederland.

DILLENBOURG, P. (2008). Integrating technologies into educational ecosystems. Distance Education, 29(2), 127-140. 
FIES, C. (2005). Classroom Response Systems: What do they add to an active learning environment? Unpublished doctoral dissertation, University of Texas at Austin, Austin, TX.

FIES, C., \& MARSHALL, J. (2006). Classroom Response Systems: A Review of the Literature. Journal of Science Education and Technology, 15(1), 101-109.

FLICK, U. (2006). An introduction to qualitative research. London: Sage Publications.

GLASER, B., \& HOLTON, J. (May de 2004). Remodeling Grounded Theory. 5,. 2, Art. 4. Retrieved the february 12, 2009, from Forum Qualitative Sozialforschung: http://www.qualitative-research.net/fqs/

GREENFIELD, P. (2009). Technology and Informal Education: What Is Taught, What Is Learned. Science 323(69) , 69-71.

GUZMAN, A. \& NUSSBAUM, M. (2009). Teaching competencies for Technology Integration in the Classroom. Journal of Computer Assisted Learning. 25, 453-469.

HOGARTH, A. (2008). Introducing a collaborative technology strategy for higher education students: Recommendations and the way forward. Educ Inf Technol., 13, 259-273.

INFANTE, C., HIDALGO, P., NUSSBAUM, M., ALARCÓN, R., \& GOTTLIEB, A. (2009), Multiple Mouse Based Collaborative One-to-One Learning, Computers and Education, (53)2, 393-401.

INFANTE, C., WEITZ, J., REYES, T., \& NUSSBAUM, M. (2009). Co-located Collaborative Learning Video Game with Single Display Groupware. Interactive Learning Environments, 99999 (1), 1-18.

KIILI, K. (2007). Foundation for problem-based gaming. British Journal of Educational Technology, 38(3), 394-404.

KIRRIEMUIR, J., \& MCFARLANE, A. (2004). Literature Review in Games and Learning, Report 8. Retrieved enero 20, 2009, from Nesta Futurelab, www.nestafuturelab.org

http://www.nestafuturelab.org/research/reviews/08_01.htm

KLOPFER, E., YOON, S. \& RIVAS, L. (2004). Comparative analysis of Palm and wearable computers for Participatory Simulations. Journal of Computer Assisted Learning, 20, 347-359.

LARREAMENDY-JOERNS, J., \& LEINHARDT, G. (2006). Going the Distance With Online Education. Review of Educational Research, 76(4), 567-605.

LIM, C. (2008). Global citizenship education, school curriculum and games: Learning Mathematics, English and Science as a global citizen. Computers \& Education 51, 1073-1093.

LINNELL, N., ANDERSON, R., FRIDLEY, J., HINCKLE, T., \& RAZMOV, V. (2007). Supporting classroom discussion with technology: A case study in 
environmental science. Proceedings Frontiers in education conference, Milwaukee F1D-4 - F1D-9.

MAYO, M. (2009). Video Games: A Route to Large-Scale STEM Education? Science 323 (79), 79-82.

MCCALLUM, E., SKINNER, C., TURNER, H., \& SAECKER, L. (2006). The TapedProblems Intervention: Increasing Multiplication Fact Fluency Using a Low-Tech, Classwide, Time-Delay Intervention. School Psychology Review, 35(3), 419-434.

MCFARLAND, D., \& Bender-deMoll, S. (2004). Classroom Structuration: A Study of Network Stabilization. Retrieved January 30, 2009, from Allacademic research: http://www.allacademic.com/meta/p110224_index.html

MEHAN, H. (1979). Learning lesson: Social organization in the classroom. Camdbrige, M.A.: Harvard University Press.

MITNIK, R., RECABARREN, M., NUSSBAUM, M., \& SOTO, A. (2009). Collaborative Robotic Instruction: A Graph Teaching Experience. Computers and Education, 53,(2), 330-342

NUSSBAUM, M., ALVAREZ, C., MCFARLANE, A., GOMEZ, F., CLARO, S., \& RADOVIC, D. (2009). Technology as small group face-to-face Collaborative Scaffolding. Computers \& Education 52(1), 147-153.

NUSSBAUM, M., ALVAREZ, C., MITNIK, R., GOMEZ, F., RADOVIC, D., CLARO, S., LOPEZ, X. \& RODRIGUEZ, P. (2008). Technology Supported Face to Face Small Group Collaborative Learning. Conference, In 16th International Conference on Computers in Education, ICCE, Taipei, Taiwan.

ORVIS, K., HORN, D. \& BELANICH, J. (2008). The roles of task difficulty and prior videogame experience on performance and motivation in instructional videogames. Computers in Human Behavior, 24 , 2415-2433.

PANITZ, T. (1999). The Case for Student Centered Instruction via Collaborative Learning Paradigms. ERIC Document Reproduction Service No. ED448444 , 13p.

PASQUALE, J., \& MEUNIER, J.-G. (2003). Categorisation techniques in computerassisted reading and analysis of texts (CARAT) in the humanities. Computers and the Humanities, 37, 111-118.

PELED, I., MERON, R., \& ROTA, S. (2007). Using a multiplicative approach to construct decimal structure. In (Eds.), J. H. Woo, H. C. Lew, K. S. Park, \& D. Y. Seo, Proceedings of the 31st Conference of the International Group for the Psychology of Mathematics Education, Vol. 4 (pp. 65-72). Seoul: PME.

PENUEL, W., BOSCARDIN, C., MASYN, K. \& CRAWFORD, V. (2007). Teaching with student response systems in elementary and secondary education settings: A survey study. Educational Technology Research and Development, 55, 315-346.

PONTEFRACT, C., \& HARDMAN, F. (2005). The Discourse of Classroom Interaction in Kenyan Primary Schools. Comparative Education, 41(1), 87-106 . 
RAVENSCROFT, A. (2007). Promoting thinking and conceptual change with digital dialogue games. Journal of Computer Assisted Learning, 23, 453-465.

RICOY, M., FELIZ, T., SEVILLANO, M. (2012). Competencias para la utilización de las herramientas digitales en la sociedad de la información. Educación XX1, 13, Disponible en: <http://espacio.uned.es/revistasuned/index.php/educacionXX1/article/view/283>. [Consulta: 03 jul. 2013]

ROBERTSON, J. \& HOWELLS, C. (2008). Computer game design: Opportunities for successful learning. Computers \& Education, 50, 559-578.

ROSAS, R., NUSSBAUM, M., CUMSILLE, P., MARIANOV, V., CORREA, M., FLORES, P., LOPEZ, X., RODRIGUEZ, P. \& SALINAS, M. (2003). Beyond Nintendo: design and assessment of educational video games for first and second grade students. Computers \& Education 40, 71-94.

ROSCHELLE, J., PENUEL, W. R., \& ABRAHAMSON, L. (2004). Classroom Response and Communication Systems: Research Review and Theory. Paper to be presented at the Annual Meeting of the American Educational Research Association (p. 8). San Diego: CA.

RULE, A., \& HALLAGAN, J. (2006). Preservice Elementary Teachers Use Drawings and Make Sets of Materials to Explain Multiplication and Division by Fractions. A research study presented at the 2nd Annual Preparing Mathematicians to Educate Teachers (PMET) Conference, Online Submission , 22, ERIC Document Reproduction Service No. ED494956) Retrieved April 7, 2009, from ERIC database.

SAMSON, P., TEASLEY, S. VAN DER PLUIJM, B., \& KNOOP, P. (2006). Using handheld PCs and peer instruction to improve science teaching and learning in higher education. International Conference on Learning Sciences, (980-981). Bloomington, Indiana.

SHAFFER, D. \& GEE, J. (2005). Before every child is left behind: How epistemic games can solve the coming crisis in education. Madison: University of WisconsinMadison.

SHAFFER, D. (2006). Epistemic frames for epistemic games. Computers \& Education, 46, 223-234.

SHECHTMAN, Z., \& LEICHTENTRITT, J. (2004). Affective teaching: a method to enhance classroom management. European Journal of Teacher Education, 27(3), 323-333.

SHERMAN, L. (2000). Postmodern Constructivist Pedagogy for Teaching and Learning Cooperatively on the Web. CyberPsychology \& Behavior, 3(1), 51-57.

SINCLAIR, J. M., \& COULTHARD, R. (1975). Towards an analysis of discourse : the English used by teachers and pupils. London : Oxford University Press. 
SUSAETA, H., JIMENEZ, F., NUSSBAUM, M., GAJARDO, I., ANDREU, J. J. \& VILLALTA, M., (2010), From MMORPG to a Classroom Multiplayer Presential Role Playing Game. Educational Technology \& Society, 13 (3), 257-269

SWAN, K., VAN HOOFT, M., KRATCOSKI, A., \& UNGER, D. (2005). Uses and Effects of Mobile Computing Devices in K-8 Classrooms. Journal of Research on Technology in Education, 38(1), 99-112.

SYH-JONG, J. (2007). A study of students' construction of science knowledge: talk and writing in a collaborative group. Educational Research, 49(1), 65-81.

THURSTON, A. (2004). Promoting multicultural education in the primary classroom: Broadband videoconferencing facilities and digital video. Computers \& Education, 43, 165-177.

VAN AMELSVOORT, M., ANDRIESSEN, J., \& KANSELAAR, G. (2007). Representational Tools in Computer-Supported Collaborative Argumentation-Based Learning: How Dyads Work With Constructed and Inspected Argumentative Diagrams. The Journal of the Learning Sciences, 16(4), 485-521.

VERMETTE, P., HARPER, L. \& DIMILLO, S. (2004). Cooperative \& Collaborative Learning....with 4-8 Year Olds: How Does Research Support Teachers' Practice? Journal of Instructional Psychology, 31(2), 130-134.

WETZEL, K., FOULGER, T. \& WILLIAMS, M. (2008). The Evolution of the Required Educational Technology Course. Journal of Computing in Teacher Education, 25(2), 67-71.

WOODWARD, J. (2006). Developing Automaticity in Multiplication Facts: Integrating Strategy Instruction with Timed Practice Drills. Learning Disability Quarterly, 29(4), 269-289.

YAN, J. (2008). Social Technology as a New Medium. The New England Journal of Higher Education, 22(4), 27-30.

ZURITA, G., \& NUSSBAUM, M. (2004a). A constructivist mobile learning environment supported by a wireless handheld network. Journal of Computer Assisted Learning, 20, 235-243.

ZURITA, G., \& NUSSBAUM, M. (2004b). Computer supported collaborative learning using wirelessly interconnected handheld computers. Computers \& Education, 42 , 289-314.

ZURITA, G., \& NUSSBAUM, M. (2007). A conceptual framework based on Activity Theory for mobile CSCL. British Journal of Educational Technology, 38,(2) , 211235.

ZURITA, G., NUSSBAUM, M., \& SHARPLES, M. (2003). Encouraging Face-to-Face Collaborative Learning through the Use of Handheld Computers in the Classroom. Lecture Notes in Computer Science, 2795, 193-208. 


\section{Correspondencia con los autores}

Marco Antonio VILLALTA PÁUCAR

Universidad de Santiago de Chile. Escuela de Psicología

Dirección postal: Avenida Ecuador 3650,

Estación Central, Santiago de Chile. Chile.

e-mail: marco.villalta@usach.cl

Teléfono: 56-27184370

\section{Angélica GUZMÁN}

Pontificia Universidad Católica de Chile. Facultad de Educación.

Dirección postal: Avenida Vicuña Mackenna 4860,

Macul, Santiago, Chile. Casilla: 7820436

e-mail:mguzmadr@puc.cl

Miguel NUSSBAUM

Pontificia Universidad Católica de Chile. Escuela de Ingeniería.

Dirección postal: Avenida Vicuña Mackenna 4860,

Macul, Santiago, Chile. Casilla: 7820436

e-mail:mn@ing.puc.cl 